Discrete Comput Geom 38:259-271 (2007)

DOI: $10.1007 / \mathrm{s} 00454-007-1337-4$

\title{
Best Partial Covering of a Convex Domain by Congruent Circles of a Given Total Area*
}

\author{
Gábor Fejes Tóth \\ Rényi Institute of Mathematics, Hungarian Academy of Sciences, \\ Pf. 127, H-1364 Budapest, Hungary \\ gfejes@renyi.hu
}

\begin{abstract}
Generalizing results of L. Fejes Tóth [3], [5], we prove the following theorem. Let $R$ be a convex domain of area $|R|$ and let $\mathcal{S}$ be a finite family of at least two congruent circles of total area $t$. Then for the area $|F|$ of the part of $R$ covered by the circles of $\mathcal{S}$, the inequality $|F|<t f(|R| / t)$ holds, where $f(x)$ is the area of the intersection of a circle of unit area and a regular hexagon of area $x$ concentric with the circle.
\end{abstract}

\section{Introduction}

We recall the classical theorem of Thue [9], [10]:

If the Euclidean plane is packed with congruent circles, then the density of the packing is at most $\pi / \sqrt{12}$.

The dual counterpart of this theorem concerning covering is due to Kershner [7]:

If the Euclidean plane is covered with congruent circles, then the density of the covering is at least $2 \pi / \sqrt{27}$.

These theorems have been generalized in various directions. Instead of congruent circles one can consider, e.g. translates of a convex disc. Now the corresponding density

* Part of this work was done during a visit of the author at the research group Theory of Combinatorial Algorithms in the Institute of Theoretical Computer Science, ETH Zürich. The visit was supported by the "Discrete and Convex Geometry" project (MTKD-CT-2005-014333) carried out by the Alfréd Rényi Institute of Mathematics, Hungarian Academy of Sciences, in the framework of the European Community's "Structuring the European Research Area" programme. The research was also supported by OTKA Grants T 038397 and T 043520 . 
bounds are the quotient of the area of the disc and the circumscribed hexagon of minimal area and the quotient of the area of the disc and the inscribed hexagon of maximal area, respectively [1], [4], [8].

The following theorems [3] are generalizations of the theorems of Thue and Kershner in another direction:

If a convex domain is packed with at least two congruent circles, then the density of the packing with respect to the domain is smaller than $\pi / \sqrt{12}$.

If a convex domain is covered with at least two congruent circles, then the density of the covering with respect to the domain is greater than $2 \pi / \sqrt{27}$.

By the density with respect to a domain we mean the total area of the circles divided by the area of the domain.

Finally we mention that the theorems of Thue and Kershner can be unified in the following general theorem [5, p. 80]:

The density of the part of the plane covered by a system of congruent circles of density $d$ is at most $d f(1 / d)$, where the function $f(x)$ is defined as the maximum of the area of the intersection of a circle of unit area and a hexagon of area $x$.

It is easy to show that the maximum of the area of the intersection is attained for a regular hexagon concentric with the circle. Thus we have the following alternative definition of the function $f(x): f(x)$ is the area of the intersection of a circle of unit area and a regular hexagon of area $x$ concentric with the circle.

In order to see that the last theorem implies the theorems of Thue and Kershner we observe that the density of the part of the plane covered by a system of circles of density $d$ is $d$ if the circles form a packing and 1 if the circles form a covering. Therefore the density of a packing of congruent circles is at most

$$
\sup _{d f(1 / d)=d} d=\pi / \sqrt{12} .
$$

Analogously, the density of a covering with congruent circles is at least

$$
\inf _{d f(1 / d)=1} d=2 \pi / \sqrt{27} .
$$

Now the question arises: Can the last theorem be generalized in the two directions mentioned above, i.e. on one hand to general convex discs instead of circles, on the other hand to a convex domain instead of the whole plane? As to the first direction, there is a theorem analogous to the last one which holds for translates of an arbitrary convex disc [2]. In what follows we are interested in a generalization in the second direction. We shall prove the following:

Theorem. Let $R$ be a convex domain and let $\mathcal{S}$ be a finite family of at least two congruent circles of total area $t$. If $F$ denotes the part of $R$ covered by the circles of $\mathcal{S}$, then

$$
|F|<t f\left(\frac{|R|}{t}\right) .
$$


Here and in what follows we use the symbol $|\cdot|$ to denote the area of a point set. The cardinality of a set $X$ will be denoted by $\|X\|$. The boundary and the convex hull of a point set $S$ in the plane will be denoted by bd $S$ and conv $S$, respectively. The distance between two points $x$ and $y$ will be denoted by $x y$.

Approximating monotone functions by step-functions, it is easy to see that our theorem implies the seemingly more general statement:

Let $R$ be a convex domain and let $P$ be a finite set of at least two points in the plane. Let $H$ be a regular hexagon of area $|H|=|R| /\|P\|$ centered at $o$. Then we have, for any non-increasing function $f$ defined for non-negative reals,

$$
\int_{R} f\left(\min _{p \in P} p x\right) d x \leq\|P\| \int_{H} f(o x) d x .
$$

For a non-decreasing function $f$ the inequality stands with the reversed sign.

In the next section we derive a formula for $|F|$ which will be used to complete the proof of the theorem in Section 3.

\section{A Formula for $|F|$}

We start by making some assumptions, which will not affect generality. First, we suppose that the circles from $\mathcal{S}$ are closed and $\mathcal{S}$ does not contain any superfluous circle, that is

(i) no circle can be omitted from $\mathcal{S}$ without decreasing the part of $R$ covered by the circles.

We also assume that

(ii) the center of each circle from $\mathcal{S}$ is contained in $R$. Furthermore, no point of $R$ has equal distance to four centers of circles from $\mathcal{S}$ and no point of bd $R$ has equal distance to three centers of circles from $\mathcal{S}$.

Finally, we assume that

(iii) the set $\bigcup_{C \in \mathcal{S}} C$ is connected and it intersects bd $R$.

Moreover,

(iv) if for a subset $\overline{\mathcal{S}}$ of $\mathcal{S},\|\mathcal{S} \backslash \overline{\mathcal{S}}\|>1$ and (bd $R) \cap\left(\bigcup_{C \in \overline{\mathcal{S}}} C\right)=\emptyset$, then the set $\bigcup_{C \in \overline{\mathcal{S}}} C$ intersects at least two of the circles $\tilde{C} \in \mathcal{S} \backslash \overline{\mathcal{S}}$.

Assumption (i) needs no justification. Also, a circle whose center lies outside $R$ can be moved so that its intersection with $R$ increases. As for the rest of assumptions (ii), it is clear that any set $\mathcal{S}$ of congruent circles can be approximated by circles satisfying these properties.

Suppose that there are two disjoint components of $\bigcup_{C \in \mathcal{S}} C$, say $\bigcup_{C \in \mathcal{S}_{1}} C$ and $\bigcup_{C \in \mathcal{S}_{2}} C$. Observe that at least one of the sets $\operatorname{conv}\left(\bigcup_{C \in \mathcal{S}_{i}} C \cap R\right), i=1$, 2, can be 
translated into the interior of $\operatorname{conv}\left(\bigcup_{C \in \mathcal{S}_{1} \cup \mathcal{S}_{2}} C \cap R\right)$. By such a translation $|F|$ does not decrease until the moved component hits some other component of $\bigcup_{C \in \mathcal{S}} C$. This shows that we can assume that $\bigcup_{C \in \mathcal{S}} C$ is connected. The assumption that $\bigcup_{C \in \mathcal{S}} C \cap$ bd $R \neq \varnothing$ is obvious.

Finally, if $\overline{\mathcal{S}}$ satisfies the conditions in (iv) and there is only a single circle $\tilde{C}$ from $\mathcal{S} \backslash \overline{\mathcal{S}}$ for which $\tilde{C} \cap\left(\bigcup_{C \in \mathcal{S} \backslash \overline{\mathcal{S}}} C\right) \neq \emptyset$, then $\bigcup_{C \in \mathcal{S} \backslash \overline{\mathcal{S}}} C$ can be brought into contact with the boundary of another circle of $\mathcal{S} \backslash \overline{\mathcal{S}}$ or the boundary of $R$ through a rotation around the center of $\tilde{C}$. Obviously, $|F|$ remains constant during this transformation.

Our formula for $|F|$ is based on the decomposition of $R$ into Dirichlet cells. The Dirichlet cell $D_{C}$ of the circle $C$ with respect to $R$ consists of those points of $R$ which are nearer to the center of $C$ than to the center of any other circle of $\mathcal{S}$. The sets $D_{C}$, $C \in \mathcal{S}$, are convex and they form, together with the complementary domain $D_{R}$ of $R$, a cell complex $\mathcal{D}$. Two cells of $\mathcal{D}$ are said to be adjacent if they have more than one boundary point in common. The connected components of the common boundary points of adjacent faces of $\mathcal{D}$ are called the edges of $\mathcal{D}$. By the above assumptions no point of the plane belongs to four cells of $\mathcal{D}$. A point belonging to the boundary of three faces of $\mathcal{D}$ is called a vertex of $\mathcal{D}$. The set of all vertices of $\mathcal{D}$ will be denoted by $\mathcal{V}$ and the set of all edges of $\mathcal{D}$ will be denoted by $\mathcal{E}$. It follows easily from Euler's theorem that

$$
\|\mathcal{V}\|=2\|\mathcal{S}\|-2 .
$$

Let $e$ be an edge of $\mathcal{D}$. We associate with $e$ a set $L(e)$ defined as follows: If $e$ is an edge belonging to adjacent Dirichlet cells $D_{C}$ and $D_{C^{*}}$ such that $C \cap C^{*} \cap e \neq \emptyset$, then let $L(e)$ be the lens $C \cap C^{*}$. If $C \cap C^{*} \cap e=\emptyset$, then let $L(e)$ be the empty set. Finally if $e$ is a component of the intersection of the boundaries of $D_{C}$ and $D_{R}$ then let $L(e)$ be that component of $C \cap D_{R}$ which contains $e$. If a vertex $v$ of $\mathcal{D}$ is the common endpoint of the edges $e_{1}, e_{2}$ and $e_{3}$ then we write

$$
I(v)=L\left(e_{1}\right) \cap L\left(e_{2}\right) \cap L\left(e_{3}\right)
$$

and

$$
u(v)=\left|L\left(e_{1}\right)\right|+\left|L\left(e_{2}\right)\right|+\left|L\left(e_{3}\right)\right|-2|I(v)| .
$$

We consider a circle $C \in \mathcal{S}$ and its Dirichlet cell $D_{C}$. Let $e_{1}, \ldots, e_{n}$ be the edges of $\mathcal{D}$ bounding $D_{C}$ in their cyclic order. Let $S_{i}$ be the segment cut off from $C$ by the line of $e_{i}$ if $e_{i} \cap C \neq \emptyset$ and $e_{i}$ belongs to the boundary of a Dirichlet cell $D_{C^{*}}, C^{*} \neq C$. Let $S_{i}=L\left(e_{i}\right)$ if $e_{i} \cap C=\emptyset$ or if $e_{i}$ belongs to the boundary of $R$. We note that if the sets $S_{i}$ and $S_{j}(i \neq j)$ have a point in common, then the parts of their boundaries which do not belong to the boundary of $C$ intersect in exactly one point. This is obvious if both sets are segments cut off from $C$ by straight lines. If however one of the sets is a component of $C \cap D_{R}$, then the statement follows from the assumption that there is no superfluous circle. In Fig. 1, e.g. the circle $C^{*}$ is superfluous. Using the last statement it is easy to verify that the intersection of $S_{i}$ and $S_{j}$ is contained in all the sets $S_{k}$ which belong to edges lying between $e_{i}$ and $e_{j}$ (or $e_{j}$ and $e_{i}$ ). According to the well-known sieve formula we have

$$
\left|\bigcup_{i=1}^{n} S_{i}\right|=\sum_{k=1}^{n}(-1)^{k-1} H_{k},
$$




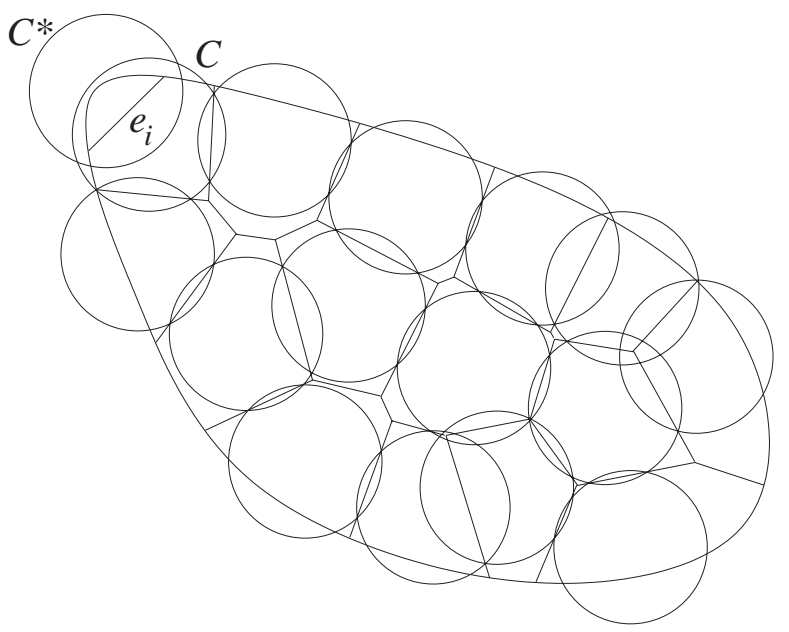

Fig. 1

where

$$
H_{k}=\sum_{1 \leq i_{1}<\cdots<i_{k} \leq n}\left|S_{i_{1}} \cap \cdots \cap S_{i_{k}}\right| .
$$

We have seen that each of the intersections $S_{i_{1}} \cap \cdots \cap S_{i_{k}}(k>2)$ is equal to the intersection of two of the sets only. On the other hand, if $e_{i}$ and $e_{j}$ are two edges of $D_{C}$ such that exactly $l(l>1)$ edges of $D_{C}$ are between them, then the area $\left|S_{i} \cap S_{j}\right|$ occurs in the sum $H_{k}\left(\begin{array}{c}l \\ k-2\end{array}\right)$ times. Thus the contribution of $\left|S_{i} \cap S_{j}\right|$ to the sum $\sum_{k=1}^{n}(-1)^{k-1} H_{k}$ is

$$
\left|S_{i} \cap S_{j}\right| \sum_{k=2}^{l+2}(-1)^{k-1}\left(\begin{array}{c}
l \\
k-2
\end{array}\right)=0 .
$$

Therefore we have

$$
\left|S_{1} \cup \cdots \cup S_{n}\right|=\left|S_{1}\right|+\cdots+\left|S_{n}\right|-\left|S_{1} \cap S_{2}\right|-\cdots-\left|S_{n} \cap S_{1}\right|
$$

and

$$
\left|C \cap D_{C}\right|=|C|-\left|S_{1}\right|-\cdots-\left|S_{n}\right|+\left|S_{1} \cap S_{2}\right|+\cdots+\left|S_{n} \cap S_{1}\right| .
$$

Summing up the corresponding equalities for all circles $C$ we obtain the relation

$$
|F|=\sum_{C \in \mathcal{S}}\left|C \cap D_{C}\right|=\sum_{C \in \mathcal{S}}|C|-\sum_{e \in \mathcal{E}}|L(e)|+\sum_{v \in \mathcal{V}}|I(v)| .
$$

Since $\sum_{c \in \mathcal{S}}|C|=t$ and $\sum_{e \in \mathcal{E}}|L(e)|-\sum_{v \in \mathcal{V}}|I(v)|=\frac{1}{2} \sum_{v \in \mathcal{V}} u(v)$, we can rewrite the last formula as follows:

$$
|F|=t-\frac{1}{2} \sum_{v \in \mathcal{V}} u(v) .
$$


In the case of covering this formula was previously established by L. Fejes Tóth (see [3] and [5, p. 69]).

Let $G$ be the part of $R$ not covered by the circles of $\mathcal{S}$. Then we have

$$
|F|=|R|-|G|
$$

Since we want to have an upper bound for $|F|$ in terms of $t$ and $|R|$, we combine formulas (2) and (3). Let $p$ and $q$ be positive numbers such that $p+q=1$. Then

$$
|F|=p t+q|R|-\frac{p}{2} \sum_{v \in \mathcal{V}} u(v)-q|G| .
$$

As the quantities $t$ and $R$ are fixed, it suffices to give a lower bound for $(p / 2) \sum_{v \in \mathcal{V}} u(V)+q|G|$.

\section{A Lower Bound for $(p / 2) \sum_{v \in \mathcal{V}} u(V)+q|G|$}

In this part of the proof it is convenient to assume that the circles of $\mathcal{S}$ have unit area, so that $t=\|\mathcal{S}\|$. Let $C_{1}, C_{2}$ and $C_{3}$ be circles of unit area centered at the vertices of a regular triangle $T$ of circumradius $r$ so that $C_{i} \cap C_{j} \neq \emptyset$ and the length of the common chord of the circles $C_{i}$ and $C_{j}$ is equal to $q r$ (Fig. 2). Let $g$ be the area of the arc-triangle enclosed by the circles $C_{1}, C_{2}$ and $C_{3}$. Write

$$
w=w(p, q)=\frac{3 p}{2}\left|C_{1} \cap C_{2}\right|+q g .
$$

We claim that

$$
\frac{p}{2} \sum_{v \in \mathcal{V}} u(v)+q|G|>2\|\mathcal{S}\| w
$$

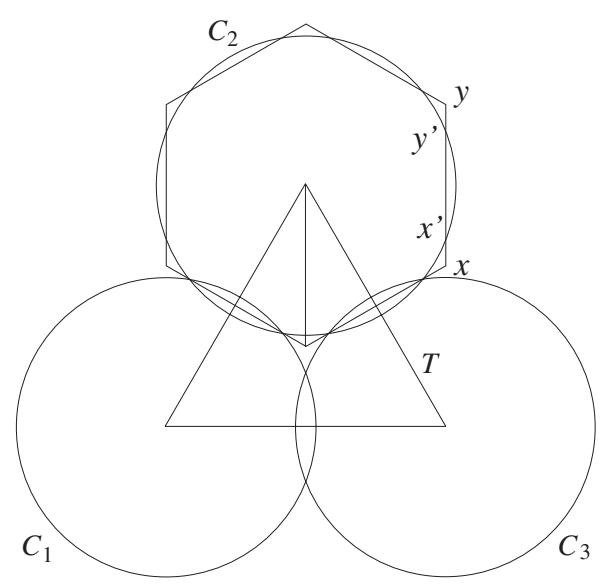

Fig. 2 
Before the proof of (5) we show that, together with the relation (4), it implies our theorem.

Let $C$ be a circle of unit area and let $H$ be a regular hexagon of area $|R| / t$ concentric with $C$. In the case when $C \subset H$ or $H \supset C$ the theorem states only the obvious inequalities $|F| \leq|R|$ and $|F| \leq t$, respectively. Therefore we suppose that the boundaries of $C$ and $H$ intersect. Let $x y$ be a side of $H$ which intersects the boundary of $C$ at the points $x^{\prime}$ and $y^{\prime}$. We choose the numbers $p$ and $q$ so that $p=1-x^{\prime} y^{\prime} / x y$ and $q=x^{\prime} y^{\prime} / x y$ and observe that with this particular choice of $p$ and $q$ the circumradius $r$ of the triangle $T$ occurring in the definition of $w$ is equal to the circumradius of $H$. Using this it is easy to see that

$$
w=\frac{1}{2}\left[p\left(1-f\left(\frac{|R|}{t}\right)\right)+q\left(\frac{|R|}{t}-f\left(\frac{|R|}{t}\right)\right)\right]=\frac{1}{2}\left(p+q \frac{|R|}{t}-f\left(\frac{|R|}{t}\right)\right) .
$$

The theorem now follows immediately from the relations (4)-(6).

It remains to prove inequality (5). We note that in inequality (5) the area of $R$ does not play a role, so we are free to change $R$ so that the $\operatorname{sum}(p / 2) \sum_{v \in \mathcal{V}} u(v)+q|G|$ decreases. Therefore we assume that

(v) $R=$ conv $\bigcup_{C \in \mathcal{S}} R \cap C$.

We start by constructing a new decomposition of $R$ as follows. We connect the centers of two circles $C_{1}$ and $C_{2}$ from $\mathcal{S}$ if the corresponding Dirichlet cells are adjacent and the common edge of the cells has a point in the interior of $C_{1} \cap C_{2}$. If $e$ is an edge of $\mathcal{D}$ common to $D_{R}$ and a circle $C \in \mathcal{S}$ such that $e \cap C \neq \emptyset$, then we connect the center of $C$ to a point of $e \cap C$. Assumptions (iii) and (iv) guarantee that these segments provide a decomposition of $R$ (Fig. 3).

This decomposition of $R$ is related to the Delone triangulation which is dual to the decomposition into Dirichlet cells. In it Delone triangles corresponding to vertices of the cell complex $\mathcal{D}$ contained in a given connected component of $G$ are glued together.

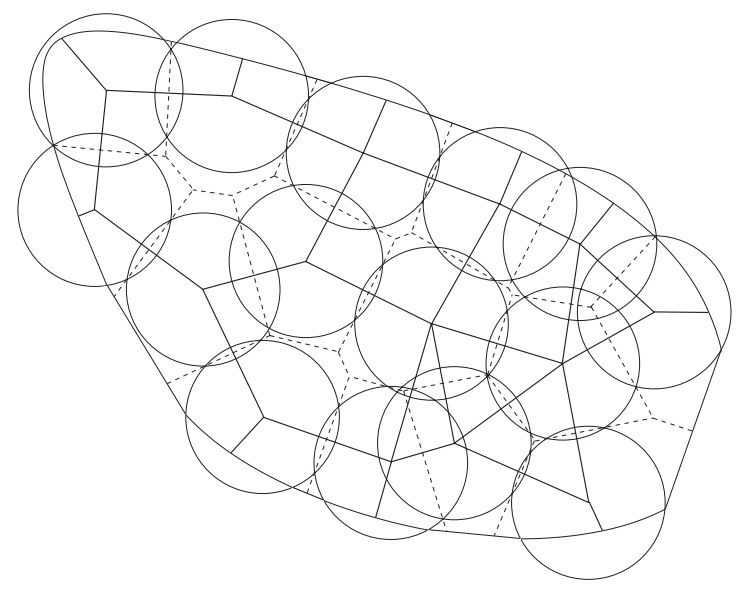

Fig. 3 
We denote the cell complex arising so by $\overline{\mathcal{D}}$ to remind us that it is related to the Delone triangulation and is dual to $\mathcal{D}$. We refer to the cells which are polygons whose vertices are centers of circles from $\mathcal{S}$ as inner cells and to the cells whose boundary contains an arc of bd $R$ as boundary cells.

We assign the vertices of $\mathcal{D}$ and the connected components of $G$ to the cells of $\overline{\mathcal{D}}$. If $\bar{D}$ is a triangular face of $\overline{\mathcal{D}}$ whose vertices are centers of circles from $\mathcal{S}$, then the center of its circumcircle is a vertex of $\mathcal{D}$ which we assign to $\bar{D}$. This vertex of $\mathcal{D}$ need not be contained in the triangle $\bar{D}$, but if it is not contained in $\bar{D}$, it is contained in the intersection of the three circles from $\mathcal{S}$ centered at the vertices of the triangle. In all other cases we assign to a cell those vertices of $\mathcal{D}$ contained in the cell which were not already assigned to a triangle.

It follows from the construction that each connected component of $G$ is contained in a cell of $\overline{\mathcal{D}}$ to which we assign it. Triangular inner cells and boundary cells with two vertices at centers of circles from $\mathcal{S}$ need not contain a component of $G$. If this is the case, we assign the empty set to them.

Let $\bar{D}_{1}, \ldots, \bar{D}_{m}$ denote the cells of $\overline{\mathcal{D}}$. Let $\mathcal{V}_{l}$ and $G_{l}, l=1, \ldots, m$, be the set of vertices of $\mathcal{D}$ and the connected component of $G$ corresponding to $\bar{D}_{l}$, respectively. We are going to estimate the sums $(p / 2) \sum_{v \in \mathcal{V}_{l}} u(v)+q\left|G_{l}\right|$ for the individual cells $\bar{D}_{l}$. Inequality (5) is an immediate consequence of the following:

Lemma. We have

$$
\frac{p}{2} \sum_{v \in \mathcal{V}_{l}} u(v)+q\left|G_{l}\right| \geq\left\|\mathcal{V}_{l}\right\| w
$$

if $\bar{D}_{l}$ is an inner cell of $\overline{\mathcal{D}}$ and

$$
\frac{p}{2} \sum_{v \in \mathcal{V}_{l}} u(v)+q\left|G_{l}\right|>\left(\left\|\mathcal{V}_{l}+1\right\|\right) w
$$

if $\bar{D}_{l}$ is a boundary cell of $\overline{\mathcal{D}}$.

Let $b$ denote the number of boundary cells of $\overline{\mathcal{D}}$. Observe that the assumption that $\mathcal{S}$ contains at least two circles implies $b \geq 2$. Thus, summing up the inequalities in the lemma for all cells of $\overline{\mathcal{D}}$ we get by (1)

$$
\frac{p}{2} \sum_{v \in \mathcal{V}} u(v)+q|G|=\sum_{l=1}^{m}\left(\frac{p}{2} \sum_{v \in \mathcal{V}_{l}} u(v)+q\left|G_{l}\right|\right)>(\|\overline{\mathcal{V}}\|+b) w \geq 2\|\mathcal{S}\| w
$$

as claimed.

For the proof of the lemma we make some further assumptions; at the same time we also generalize the conditions.

It is easy to see that if $\mathcal{V}_{l}$ consists of a single vertex $v$ and $G_{l}=\emptyset$, then $u(v)$ can be decreased. Therefore, we assume that

(vi) $G_{l} \neq \emptyset$ for $l=1, \ldots, m$.

If $\bar{D}_{l}$ is a boundary cell, then assumption (v) implies that bd $G_{l} \cap$ bd $R$ is a line segment. Substituting $R$ by its supporting half-plane containing this segment, $\sum_{v \in \mathcal{V}_{l}} u(v)$ does 


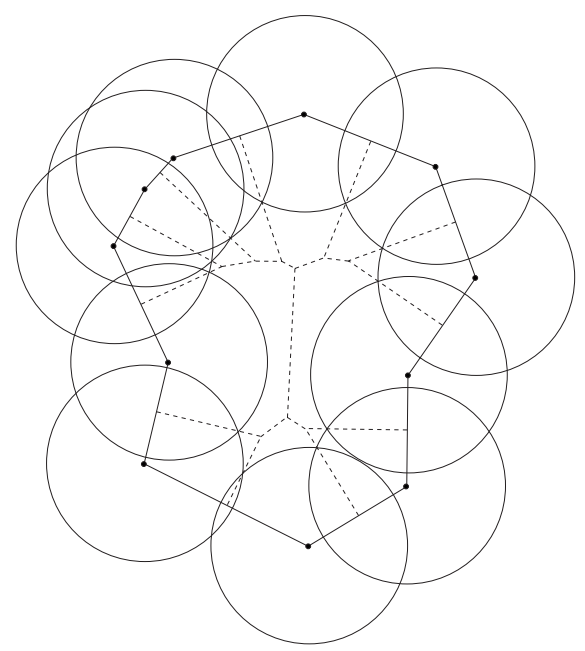

Fig. 4

not increase. Thus, it suffices to consider the case when

(vii) $R$ is a half-plane.

For such a boundary cell there are two segments bounding $\bar{D}_{l}$ connecting centers of circles from $\mathcal{S}$ to boundary points of $R$. We assume that these segments are perpendicular to the line bounding $R$.

Let $C_{1}, \ldots, C_{n}$ be the circles from $\mathcal{S}$ centered at the vertices of an inner cell $\bar{D}_{l}$ of $\overline{\mathcal{D}}$ enumerated in their cyclic order as their centers follow on bd $\bar{D}_{l}$. Observe that, by assumption (iv), each of these circles has a single arc common with bd $G_{l}$ and these arcs have the same cyclic order on bd $G_{l}$ as the centers of the circles on bd $\bar{D}_{l}$ (Fig. 4). It is easy to verify that

$$
\left\|\mathcal{V}_{l}\right\|=n-2
$$

and

$$
\sum_{v \in \mathcal{V}_{l}} u(v)=\left|C_{1} \cap C_{2}\right|+\left|C_{2} \cap C_{3}\right|+\cdots+\left|C_{n} \cap C_{1}\right|
$$

Consider now a boundary cell $\bar{D}_{l}$ of $\overline{\mathcal{D}}$. In order to unify the picture we consider the complement of $R$, a half-plane by assumption (vii), to be a circle of infinite radius centered at the point at infinity corresponding to the direction perpendicular to the line bounding $R$. Then (7) and (8) remain valid if we allow one of the circles $C_{1}, \ldots, C_{n}$ to be the complement of $R$ (Fig. 5).

In our following considerations we treat inner and boundary cells together. We assume that the notation is chosen so that, for boundary cells, $C_{1}$ denotes the complement of $R$. We write $C_{i+n}=C_{i}$. Let $o_{i}$ denote the center of $C_{i}$. For a boundary cell let $\underline{o}_{1}$ be the point of intersection of $o_{1} o_{2}$ and bd $C_{1}$ and let $\bar{o}_{1}$ be the point of intersection of $o_{n} o_{1}$ and bd $C_{1}$. Let $A_{i}$ denote the arc bd $C_{i} \cap$ bd $G_{l}, i=1, \ldots, n$. We are looking for the 


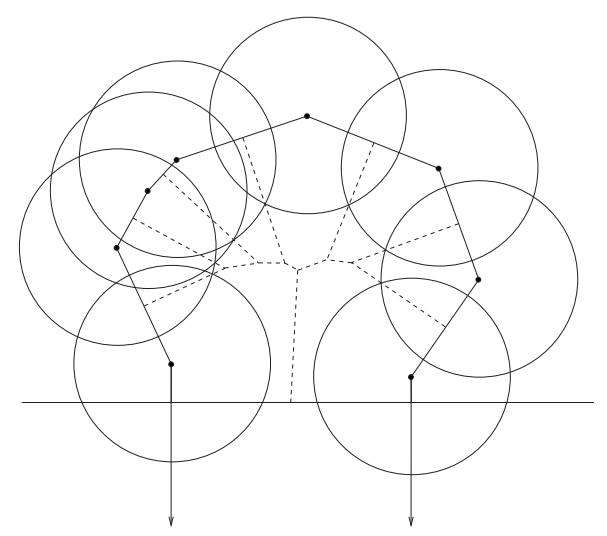

Fig. 5

minimum value of

$$
s=\frac{p}{2} \sum_{v \in \mathcal{V}_{l}} u(v)+q\left|G_{l}\right|,
$$

while the circles $C_{1}, \ldots, C_{n}$ vary under the following conditions:

(a) $C_{i} \cap C_{i+1} \neq \emptyset$ for $i=1, \ldots, n$.

(b) $0 \leq \angle o_{i-1} o_{i} o_{i+1} \leq \pi$.

(c) We allow the polygonal region $o_{1}, \ldots, o_{n}$ to overlap itself, however consider the overlapping parts to be in separate sheets and calculate the area of $G_{l}$ accordingly. As long as non-neighboring arcs do not intersect, we calculate $\sum_{v \in \mathcal{V}_{l}} u(v)$ according to formula (8) even when some vertices from $\mathcal{V}_{l}$ are not trivalent.

(d) If two non-neighboring arcs $A_{i}$ and $A_{j}$ intersect in a point, then we divide $\bar{D}_{l}$ with the segment or ray $o_{i} o_{j}$ into two parts, vary from there on the circles $C_{i}, C_{i+1}, \ldots, C_{j}$ and $C_{j}, C_{j+1}, \ldots, C_{i}$ separately under conditions (a)-(c), and calculate s as the sum of the respective values for the two components. We iterate step (d) if a new pair of non-neighboring arcs intersect in a point.

It is easy to see that $s$ attains its minimum under these conditions. We are going to show that if $n \geq 4$ then $s$ can be decreased, therefore the minimum is attained for $\left\|\mathcal{V}_{l}\right\|=n-2$ separate "triangles".

Small rotations of the circle $C_{i}$ around the midpoint of the arc $A_{i}$ show that $s$ can be decreased unless the arcs $C_{i-1} \cap \mathrm{bd} C_{i}$ and $C_{i+1} \cap \mathrm{bd} C_{i}$ have equal lengths. In particular, we may assume that

$$
o_{1} o_{2}=o_{2} o_{3}=\cdots=o_{n} o_{1} \quad \text { for inner cells }
$$

and

$$
2 \underline{o}_{1} o_{2}=o_{2} o_{3}=\cdots=2 \bar{o}_{n} o_{1} \quad \text { for boundary cells. }
$$


Suppose that $C_{i}$ touches $C_{i+1}$ for all $i=1, \ldots, n$. Then applying a homothety of ratio $1-\varepsilon, \varepsilon>0$, to the set $\left\{o_{1}, \ldots, o_{n}\right\}$ the area $\left|G_{l}\right|$ decreases in the order $\varepsilon$, while the sum $\left|C_{1} \cap C_{2}\right|+\left|C_{2} \cap C_{3}\right|+\cdots+\left|C_{n} \cap C_{1}\right|$ increases in the order $\varepsilon^{2}$, showing that in the case yielding the minimum of $s$, the interiors of consecutive circles intersect.

Next observe that we have to deal only with the case when all angles $\angle o_{i-1} o_{i} o_{i+1}$ are convex. For if $\angle o_{i-1} o_{i} o_{i+1} \geq \pi$, then $C_{i}$ can be moved so that $\left|G_{l}\right|$, as well as $\left|C_{i-1} \cap C_{i}\right|$ and $\left|C_{i} \cap C_{i+1}\right|$, decreases.

Let $S_{i}$ be the sector of $C_{i}$ cut out by $\angle o_{i-1} o_{i} o_{i+1}$. Write $\bar{S}_{i}=S_{i} \cap \bar{D}_{l}$. If $\bar{D}_{l}$ is convex, then the arcs $\bar{D}_{l} \cap$ bd $C_{i}$ and $\bar{D}_{l} \cap$ bd $C_{j}, i \neq j$, intersect in at most one point. It follows that $\bar{S}_{i} \cap \bar{S}_{j}$ is contained in all sets $\bar{S}_{k}$ between $\bar{S}_{i}$ and $\bar{S}_{j}$ or $\bar{S}_{j}$ and $\bar{S}_{i}$ in the cyclic order. Hence we get, in the same way as in the derivation of formula (2), that

$$
\left|\bigcup_{i=1}^{n} \bar{S}_{i}\right|=\sum_{i=1}^{n}\left|\bar{S}_{i}\right|-\sum_{i=1}^{n}\left|\bar{S}_{i} \cap \bar{S}_{i+1}\right| .
$$

Let $G_{l}$ be an inner cell. Then

$$
\begin{aligned}
\left|\bigcup_{i=1}^{n} \bar{S}_{i}\right| & =\sum_{i=1}^{n}\left|\bar{S}_{i}\right|-\sum_{i=1}^{n}\left|\bar{S}_{i} \cap \bar{S}_{i+1}\right|+\sum_{i=1}^{n}\left|S_{i} \backslash \bar{D}_{l}\right|-\sum_{i=1}^{n}\left|S_{i} \backslash \bar{D}_{l}\right| \\
& =\sum_{i=1}^{n}\left|S_{i}\right|-\frac{1}{2} \sum_{i=1}^{n}\left|C_{i} \cap C_{i+1}\right| .
\end{aligned}
$$

Since $\sum_{i=1}^{n}\left|S_{i}\right|=(n-2) / 2$, we have

$$
\left|G_{l}\right|=\left|\bar{D}_{l}\right|-\left|\bigcup_{i=1}^{n} \bar{S}_{i}\right|=\left|\bar{D}_{l}\right|-\frac{1}{2}\left(n-2+\sum_{i=1}^{n}\left|C_{i} \cap C_{i+1}\right|\right) .
$$

Hence, in view of (8), we get

$$
s=\frac{1}{2} \sum_{i=1}^{n}\left|C_{i} \cap C_{i+1}\right|+q\left(\left|\bar{D}_{l}\right|-\frac{n-2}{2}\right) .
$$

It is easy to check that (10) holds for boundary cells as well.

We recall a result by Habicht and van der Waerden [6]. Given a convex quadrilateral with three sides of equal length at most as long as the fourth side, the area of the quadrilateral can be decreased by keeping the longest side fixed and moving the opposite side so that the length of all sides remain constant. In view of (9)-(11) this implies that $s$ can be decreased if $\bar{D}_{l}$ is an inner cell and $n \geq 4$ or it is a boundary cell and $n \geq 5$.

The case of a boundary cell with $n=4$ has to be treated separately. Let $\underline{o}_{1} o_{1}=a$ and $\underline{o}_{1} \bar{o}_{1}=2 x$ (Fig. 6). Keeping in mind (10), we observe that, for $x=\sqrt{3 a^{2}+2 \sqrt{\pi} a-\pi}$, $C_{1}$ and $C_{3}$ touch and, for $x=\sqrt{\pi}, C_{2}$ and $C_{4}$ touch. We have $\left|\bar{D}_{l}\right|=x\left(2 a+\sqrt{4 a^{2}-x^{2}}\right)$. The function $x\left(2 a+\sqrt{4 a^{2}-x^{2}}\right)$ takes its maximum at $x=\sqrt{3} a$. Depending on whether $\sqrt{3 a^{2}+2 \sqrt{\pi} a-\pi}<x<\sqrt{3} a$ or $\sqrt{3} a<x \leq \sqrt{3 a^{2}+2 \sqrt{\pi} a-\pi}$ the area $\left|\bar{D}_{l}\right|$, as well as $s$, decreases by keeping the distances $\underline{o}_{1} o_{1}, o_{1} o_{2}, o_{2} o_{3}, o_{3} o_{4}$ and $o_{4} \bar{o}_{1}$ fixed and decreasing, respectively increasing $x$, until $C_{2}$ and $C_{4}$ or $C_{1}$ and $C_{3}$ touch. 


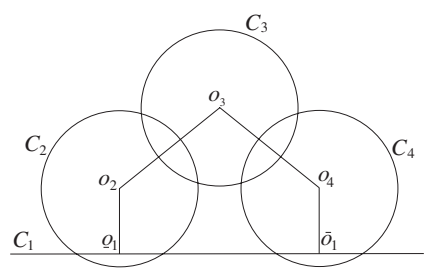

Fig. 6

Recapitulating, if $n \geq 4$ then $s$ can be decreased by appropriately moving the circles $C_{1}, \ldots, C_{n}$ until two non-neighboring arcs $A_{i}$ and $A_{j}$ intersect in a point, so that $\bar{D}_{l}$ can be broken into two smaller cells. Repeating this process we reach a situation with "triangular cells" only. Observe that at least one of the "descendants" of a boundary cell will be a boundary cell as well.

Now consider the case $n=3$. It is easy to see that if $C_{1}, C_{2}, C_{3}$ are circles of finite radius, then $s \geq w$. If, on the other hand, $C_{1}$ is a half-plane, then the minimum of $s$ is attained when the line bounding it intersects the circles $C_{2}$ and $C_{3}$ in $u_{1}$ and $v_{1}$ and $u_{2}$ and $v_{2}$, respectively, so that $u_{1} v_{1}=u_{2} v_{2}=x y=(q / p) v_{1} u_{2}$, where $x y$ is the common chord of the two circles (Fig. 7). We denote this minimum value by $w^{*}=w^{*}(p, q)$. The lemma will follow by showing that $w^{*}>2 w$.

An easy calculation yields that

$$
w=\frac{3}{\pi} \arctan \frac{q}{\sqrt{3}}-\frac{q}{2}
$$

and

$$
w^{*}=\frac{2}{\pi} \arctan q-\frac{q}{2}
$$

It can be checked that

$$
2 \arctan q-\frac{q \pi}{2}-2\left(3 \arctan \frac{q}{\sqrt{3}}-\frac{q \pi}{2}\right)=2\left(\arctan q+\frac{q \pi}{4}-3 \arctan \frac{q}{\sqrt{3}}\right)
$$

takes the value zero for $q=0$ and $q=1$ and has a single maximum for $0<q<1$. Therefore we have $w^{*}>2 w$, as claimed. This completes the proof of the lemma, as well as the proof of the theorem.

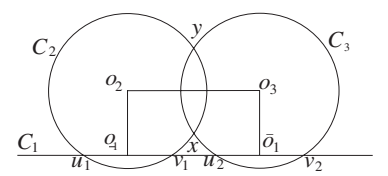

Fig. 7 


\section{References}

1. Bambah, R. P., and Rogers, C. A., Covering the plane with convex sets, J. London Math. Soc. 27 (1952), 224-229.

2. Fejes Tóth, G., Covering the plane by convex discs, Acta Math. Acad. Sci. Hungar. 23 (1972), 263-270.

3. Fejes Tóth, L., Über dichteste Kreislagerung und dünnste Kreisüberdeckung, Comment. Math. Helvetici 23 (1949), 342-349.

4. Fejes Tóth, L., Some packing and covering theorems, Acta Univ. Szeged Acta Sci. Math. 12/A (1950), 62-67.

5. Fejes Tóth, L., Lagerungen in der Ebene auf der Kugel und im Raum (zweite Auflage), Springer-Verlag Berlin, 1972.

6. Habicht, W., and van der Waerden, B. L., Lagerungen von Punkten auf der Kugel, Math. Ann. 123 (1951), 223-234.

7. Kershner, R., The number of circles covering a set, Amer. J. Math. 61 (1939), 665-671.

8. Rogers, C. A., The closest packing of convex two-dimensional domains, Acta Math. 86 (1951), 309-321.

9. Thue, A., Om nogle geometrisk-taltheoretiske theoremer, Forhdl. Skand Naturforsk. 14 (1892), 352-353.

10. Thue, A., Über die dichteste Zusammenstellung von kongruenten Kreisen in der Ebene, Chriatiania Vid. Selsk. Skr. 1 (1910), 3-9.

Received July 10, 2006, and in revised form September 18, 2006. Online publication July 17, 2007. 